\title{
Prediction of pandemic influenza
}

\author{
Hiroshi Nishiura
}

Received: 26 April 2011/Accepted: 10 June 2011/Published online: 17 July 2011

(C) The Author(s) 2011. This article is published with open access at Springerlink.com

Nougairède et al. [1] critically discussed adverse effects of epidemic modeling in having wrongly advised devastating consequences of a pandemic prior to the actual 2009 pandemic. Especially, the authors emphasized incapability of models to yield correct prediction. As one of modeling experts, I have concerns regarding their interpretations.

First, it should be remembered that prediction has two different components: projection and forecasting [2]. A projection is an attempt to describe what would happen under certain assumptions and hypotheses, while a forecast is a quantitative attempt to predict what will happen in the future [3]. Prior to the 2009 pandemic, modeling studies offered projections with 'what if' scenarios under various hypotheses of public health interventions (e.g. [4, 5]). It is clear that those studies did not intend to offer quantitatively valid forecasts. If projections and forecasts were mixed up in policymaking (i.e. if the projected numbers seriously

Commenting on: Nougairède A, Charrel RN, Raoult D. Models cannot predict future outbreaks: A/H1N1 virus, the paradigm. Eur J Epidemiol 2011;26:183-186.

H. Nishiura $(\bowtie)$

School of Public Health, The University of Hong Kong, Level 6, Core F, Cyberport 3, 100 Cyberport Road, Pokfulam,

Hong Kong

e-mail: nishiura@hku.hk

H. Nishiura

PRESTO, Japan Science and Technology Agency, Saitama, Japan influenced policymaking as if they were forecasts), any troubles in relevant policy should not be attributed to modeling studies, but rather, to communications between experts and policymakers.

Second, any prediction effort requires a baseline input of the 'pandemic in our mind', and empirical data of past pandemics in the twentieth century were almost exclusively available objective references. Unfortunately, absence of pandemics for longer than 40 years forced us to focus on the historical data, and such a focus led us to offer the 'worst case' scenarios. Given that cases infected with highly pathogenic avian influenza (H5N1) are continuously reported, always keeping the worst case scenario in our mind is not necessarily bad. However, the foregoing worst case scenario was misleading for health policy in many circumstances from 2009 to 2010, and a specific lesson for modeling is that we have to constantly consider the point of variations in pandemic potential (e.g. consider wide variability in the severity).

Third, there were two specific lessons for modeling in addition to biased emphasis on the worst case scenarios. Namely, (1) model structure and assumptions were not consistent with field observation, which is represented by difficulties in ascertaining all influenza cases and estimating the case fatality ratio [6,7], and (2) real-time estimation was not incorporated into pandemic preparedness plans, and thus, essential data needs for such real-time exercises were not systematically considered prior to the pandemic.

In future, similar misunderstanding could be avoided by addressing the above mentioned issues. Rather than regarding criticisms of Nougairède et al. [1] as an abandonment of prediction science, revised pandemic plans should be formulated by experimentally solving problems that have been seen from 2009 . 


\section{Acknowledgments}

HN received financial support from the Japan Science and Technology Agency PRESTO program.

\section{References}

1. Nougairède A, Charrel RN, Raoult D. Models cannot predict future outbreaks: A/H1N1 virus, the paradigm. Eur J Epidemiol. 2011;26:183-86.

2. Keyfitz N. On future population. J Am Stat Assoc. 1972;67:347-63.

3. Nishiura H. Real-time forecasting of an epidemic using a discrete time stochastic model: a case study of pandemic influenza (H1N1-2009). Biomed Eng Online 2011;10:15.

4. Wu JT, Riley S, Fraser C, Leung GM. Reducing the impact of the next influenza pandemic using household-based public health interventions. PLoS Med. 2006;3:e361.

5. Ferguson NM, Cummings DA, Fraser C, Cajka JC, Cooley PC, Burke DS. Strategies for mitigating an influenza pandemic. Nature 2006;442:448-52.

6. Nishiura H. Case fatality ratio of pandemic influenza. Lancet Infect Dis. 2010;10:443-4.

7. Nishiura H. Joint quantification of transmission dynamics and diagnostic accuracy applied to influenza. Math Biosci Eng. 2011;8:49-64.

\section{Prof Raoult replies}

I thank H. Nishiura [1] who confirms that the past inability of models to predict any feature of H1N1 pandemic [2] was caused by gaps of knowledge. This is my basic point, the gap of knowledge is much too large to propose any suitable model, in any outbreak, as by definition these are stochastic events. For example, it is clear that microorganisms interfer with others and that this is modifying host susceptibility.
We cannot predict the other microbes circulating during an outbreak limiting our understanding. The protective effect of past ignored outbreaks such as for H1N1 cannot be measured [3]. Moreover human societies learn from past outbreaks. Then they are modifying their habits and are defying predictors [4]. Finally the worst predicting model arrogance was reached when the modeling of the future the outbreak of H5N1, (a non human pathogen!) was proposed based on surrealistic assumptions (transmission ratio, fatality rates, source of the outbreak)! These models terrified policy makers and excited media. As a consequence the future pandemic was prepared as a war! This pushed extreme fears that were not confirmed, and undermine the credibility not only of the predictors (that does not hurt me) but also of the all profession of microbiologist. In fact model based predictions are looking like science but as much as astrology is looking like astronomy. Finally I would like to assure Dr H. Nishiura of my compassion, as he is living in a country facing huge earthquakes, unpredicted despite a lot of geologist predictors!

\section{References}

1. Nishiura H. Prediction of pandemic influenza. Eur $\mathbf{J}$ Epidemiol 2011; In press.

2. Nougairede A, Charrel RN, Raoult D. Models cannot predict future outbreaks: A/H1N1 virus, the paradigm. Eur J Epidemiol 2011 March;26(3):183-6.

3. Raoult D. Microbe interactions undermine predictions. Science 2011 January 14;331(6014):144-5.

4. Raoult D. Moleculalr, epidemiological, and clinical complexities of prediction patterns of infectious diseases. Frontiers in cellular and infection microbiology 2011;2(25):1-2.

Open Access This article is distributed under the terms of the Creative Commons Attribution Noncommercial License which permits any noncommercial use, distribution, and reproduction in any medium, provided the original author(s) and source are credited. 